\section{Müde Schnarcher}

Pneumologen um Dr. Or Kalchiem-Dekel fanden heraus, dass schnarchende Schläfer tagsüber umso müder sind, je lauter sie nachts tönen. Die Ärzte bestimmten den Schalldruck der nächtlichen akustischen Aussonderungen. Im Schnitt erreichten die Schnarcher einen Pegel von 46 Dezibel $(\mathrm{dB})$, was dem einer Unterhaltung in normaler Lautstärke in einem Meter Entfernung entspricht. Männer unterschieden sich nicht von Frauen. Manche schafften aber auch knapp $65 \mathrm{~dB}$ - ähnlich laut wie eine $10 \mathrm{~m}$ entfernte Straße. Mit dem Lärm stieg auch die Tagesmüdigkeit an. Interessanterweise waren Männer trotz ähnlicher Lautstärke deutlich häufiger müde.

Kalchiem-Dekel O et al. Laryngoscope 2016 (online first)

\section{"Viagra für Frauen" hat kaum Effekt}

Der in den USA seit ca. 6 Monaten erhältliche Wirkstoff Flibanserin (Handelsname Addyi $^{\mathrm{TM}}$ ) hat Studien zufolge kaum Wirkung. Nebenwirkungen hingegen lassen nicht lang auf sich warten. Wissenschaftler aus den Niederlanden und Belgien werteten Daten von fünf veröffentlichten und drei unveröffentlichten Studien aus. 5914 Frauen nahmen insgesamt teil. Häufig traten Schwindel, Schläfrigkeit, Übelkeit und Erschöpfung auf, allerdings meist in milder Form. Durchschnittlich hatten die Frauen in zwei Monaten durch die Pille eine befriedigenden sexuellen Erfahrung mehr.

Ärzte Zeitung, 3.3.2016

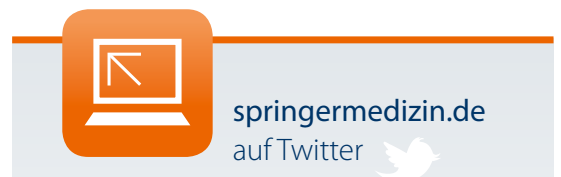

Der Frühling kommt!!!

Und wir zwitschern ganz laut mit "lauschen" Sie uns und werden Sie zum „Follower"

- twitter.com/springermedizin

Eine Frage der Ethik:

\title{
Abschalten ist keine aktive Sterbehilfe
}

Juristisch ist es wenig bedeutsam, ob eine lebenserhaltende Maßnahme beendet oder gar nicht erst begonnen wird.

Laut Professor Frank Joachim Erbguth vom Klinikum Nürnberg sei noch immer der Irrtum weit verbreitet, das Abschalten der Beatmung, eines Herzschrittmachers oder das Entfernen der PEG-Sonde sei aktive Sterbehilfe. Erbguth verwies auf den Fall Küllmer-Putz, der vor Jahren Aufsehen erregte: Ein Pflegeheim verweigerte einer Komapatientin den Abbruch der künstlichen Ernährung. Die Kinder bestanden darauf, weil es dem Willen der Patientin entsprach. Ihr Anwalt riet ihnen, den PEG-Schlauch zu durchtrennen, was sie auch taten.
Daraufhin wurde der Anwalt wegen versuchten Totschlags vom Landgericht verurteilt. Der Bundesgerichtshof (BGH) hob das Urteil jedoch auf und stellte klar: Das Beenden einer Therapie kann sowohl durch Unterlassen als auch durch aktives Handeln geschehen. Wird eine Therapie aktiv abgebrochen, ist dies keine Tötung. Mit diesem Urteil schaffte der BGH endlich Rechtssicherheit. In der Praxis, so Erbguth, vermeiden es Ärzte und Angehörige aber noch immer, den Ausknopf zu drücken. Er rät allen beteiligten Ärzten, Behandlungen und die etwaige Beendigung gut $\mathrm{zu}$ dokumentieren, um sich nicht angreifbar zu machen.

\section{Wenn SSRI und SNRI versagen}

\section{Opioide für Depressive}

In einer Studie sprachen bis zu 50\% der an therapieresistenter Depression erkrankten Patienten auf eine Buprenorphin/Samidorphan-Kombi an.

Die Opioidtherapie bei Gemütskranken könnte eine Renaissance erleben. Am geeignetesten scheint Buprenorphin wegen der geringen atemdepressiven Effekte zu sein. Zusammen mit $\mu$-Rezeptor-Antagonisten, um das Suchtpotenzial zu senken, könnten daraus nützliche Antidepressiva entstehen. Denn 40\% der Erkrankten sprechen nicht genügend auf klassische Anti depressiva an, wie Ärzte um Dr. Maurizio Fava von der Harvard Medical School in Boston berichten. In einer vor Kurzem veröffentlichten Studie konnte eine niedrigdosierte Buprenorphinbehandlung zudem Suizidgedanken abschwächen. Sein Team untersuchte den Ansatz in einem Versuch mit 142 Depressiven. Sie erhielten entweder ein Placebo oder Buprenorphin und Sami- dorphan ( $\mu$-Antagonist) in gleicher Dosierung $(2 \mathrm{mg} / 2 \mathrm{mg}$ oder $8 \mathrm{mg} / 8 \mathrm{mg})$. $\mathrm{Zu} \mathrm{Be}-$ ginn lag der Score auf der Hamilton-Depressionsskala im Schnitt bei 23 Punkten (mittlere/schwere Depression). Nach vier Wochen und einer Woche Ausschleichen sank er unter Placebo um 7,3 Punkte, unter der 2 mg Dosierung um 9,3 Punkte und unter höherer Dosis um 6,4 Punkte. Es ergab sich unter der niedrigen Dosis ein signifikanter Vorteil von 2,8 Punkten im Vergleich zum Placebo. Nicht signifikant war der Vorteil von 0,5 Punkten zur höheren Dosis. Die Therapie erwies sich als ähnlich effektiv wie die Augmentation mit Antipsychotika. Der Ansatz sollte laut den Forschern daher weiter untersucht werden, dabei müsse geklärt werden, wie lange die Therapieeffekte anhalten und ob der $\mu$-Antagonist tatsächlich eine Suchtentwicklung verhindert.

(mut)

Fava Met al. Am J Psychiat 2016 (online first) 